\title{
ASYMPTOTIC NORMALITY OF DISCRETE-TIME MARKOV CONTROL PROCESSES
}

\author{
ARMANDO F. MENDOZA-PÉREZ, ${ }^{*}$ Universidad Politécnica de Chiapas \\ ONÉSIMO HERNÁNDEZ-LERMA, ${ }^{* *}$ CINVESTAV
}

\begin{abstract}
In this paper we study the asymptotic normality of discrete-time Markov control processes in Borel spaces, with possibly unbounded cost. Under suitable hypotheses, we show that the cost sequence is asymptotically normal. As a special case, we obtain a central limit theorem for (noncontrolled) Markov chains.
\end{abstract}

Keywords: (Discrete-time) Markov control process; average cost criteria; average variance; asymptotic normality

2010 Mathematics Subject Classification: Primary 93E20; 90C40; 60F05; 60J05

\section{Introduction.}

In this paper we are concerned with discrete-time Markov control processes (MCPs) in Borel spaces, with the long-run pathwise average cost

$$
S(\varphi, x):=\limsup _{n \rightarrow \infty} \frac{1}{n} S_{n}(\varphi, x)
$$

where

$$
S_{n}(\varphi, x):=\sum_{k=0}^{n-1} C_{\varphi}\left(x_{k}\right) .
$$

Here, $\varphi$ denotes the control policy being used (see Section 2 for details), $\left\{x_{k}\right\}$ is the controlled state (Markov) process, and $x_{0}=x$ is the initial state. The underlying optimal control problem is to minimize (almost surely) $S(\varphi, x)$ over the family of admissible control policies $\varphi$ for every $x$. To analyze some aspects of this problem, it is important to determine the asymptotic behavior of the cost sequence $S_{n}(\varphi, x)$. In particular, if it can be ensured that it is asymptotically normal then we can deduce important optimality properties, which are particularly useful when the MCP depends on unknown parameters [13], [15].

In this paper we give reasonably mild conditions for the cost sequence $\left\{S_{n}(\varphi, x)\right\}$ to be asymptotically normal. This line of research was initiated by Mandl [14], and further extended by himself and his associates [11], [12], [13], [15] for MCPs with a finite state space. MendozaPérez [17], [16] extended the results in [14] to discrete-time MCPs with Borel spaces. Other extensions include results by Lánská [9] for a class of controlled diffusions, and by PrietoRumeau and Hernández-Lerma [20] for continuous-time MCPs with a countable state space.

Received 11 March 2010; revision received 14 May 2010.

* Postal address: Universidad Politécnica de Chiapas, Calle Eduardo J. Selvas S/N, Tuxtla Gutiérrez, Chiapas, Mexico. Email address: mepa680127@hotmail.com

** Postal address: Department of Mathematics, CINVESTAV-IPN, A. Postal 14-740, Mexico DF 07000, Mexico.

Email address: ohernand@math.cinvestav.mx

Research partially supported by CONACyT grant 104001 . 
In all of these references $\varphi$ is assumed to be a deterministic and optimal policy. In contrast, here we obtain asymptotic normality for every randomized stationary policy $\varphi$ (in a given set $\Phi$-see Section 2) which may be neither deterministic nor optimal. This means that our main result, Theorem 3.1, yields in particular a central limit theorem for noncontrolled Markov chains. On the other hand, since we allow randomized policies $\varphi$, our results are applicable to constrained MDPs [17], [18], and to Markov games [5]. Moreover, it is worth noting that the existing proofs of the central limit theorem for Markov chains require strong ergodicity conditions, for instance, geometric ergodicity [19, Theorem 17.0.1] or polynomial ergodicity [8, Theorems 4.1, 4.2, 4.3, and 4.4]. Here, however, instead of an ergodicity requirement we impose a Lyapunov-like condition (see Assumption 3.2), in addition to growth and continuity-compactness conditions (Assumptions 3.1 and 3.3).

To obtain our results, we combine two approaches. The first approach is to show, under suitable conditions, the existence of solutions to the Poisson equation; see (3.4) below. The second approach is Mandl's approach [14] using characteristic functions.

The remainder of the paper is organized as follows. Section 2 contains a brief description of the Markov control model of interest. In Section 3 we introduce our hypotheses and state our main result, Theorem 3.1, which is proved in Section 4. Finally, a linear system/quadratic cost (or LQ system) in Section 5 illustrates our results.

\section{The control model}

Let $(\mathbb{X}, \mathbb{A},\{A(x): x \in \mathbb{X}\}, Q, C)$ be a discrete-time Markov control model with state space $\mathbb{X}$ and control (or action) set $\mathbb{A}$, both assumed to be Borel spaces with $\sigma$-algebras $\mathbb{B}(\mathbb{X})$ and $\mathscr{B}(\mathbb{A})$, respectively. For each $x \in \mathbb{X}$, there is a nonempty Borel set $A(x)$ in $\mathscr{B}(\mathbb{A})$ which represents the set of feasible actions in the state $x$. The set

$$
\mathbb{K}:=\{(x, a): x \in \mathbb{X}, a \in A(x)\}
$$

is assumed to be a Borel subset of $\mathbb{X} \times \mathbb{A}$. The transition law $Q$ is a stochastic kernel on $\mathbb{X}$ given $\mathbb{K}$ and the one-stage cost $C$ is a real-valued measurable function on $\mathbb{K}$.

Let $\Phi$ be the set of all stochastic kernels $\varphi$ on $A$ given $\mathbb{X}$ for which $\varphi(A(x) \mid x)=1$ for all $x \in \mathbb{X}$.

\subsection{Control policies}

For every $n=0,1, \ldots$, let $\mathbb{H}_{n}$ be the family of admissible histories up to time $n$; that is, $\mathbb{H}_{0}:=\mathbb{X}$ and $\mathbb{H}_{n}:=\mathbb{K}^{n} \times \mathbb{X}$ if $n \geq 1$. A control policy is a sequence $\pi=\left\{\pi_{n}\right\}$ of stochastic kernels $\pi_{n}$ on $\mathbb{A}$ given $\mathbb{H}_{n}$ such that $\pi_{n}\left(A\left(x_{n}\right) \mid h_{n}\right)=1$ for every $n$ history $h_{n}=\left(x_{0}, a_{0}, \ldots, x_{n-1}, a_{n-1}, x_{n}\right)$ in $\mathbb{H}_{n}$. The class of all policies is denoted by $\Pi$.

A policy $\pi=\left\{\pi_{n}\right\}$ is said to be a (randomized) stationary policy if there exists a stochastic kernel $\varphi \in \Phi$ such that $\pi_{n}\left(\cdot \mid h_{n}\right)=\varphi\left(\cdot \mid x_{n}\right)$ for all $h_{n} \in H_{n}, n=0,1, \ldots$. As usual, we identify $\Phi$, the set of stochastic kernels on $A$ given $\mathbb{X}$, with the set of all randomized stationary policies. Hence, $\Phi \subset \Pi$.

If $\pi=\{\varphi\}$ is a stationary policy, abusing the notation, we write $\pi=\varphi$.

For notational ease, we write

$$
C_{\varphi}(x):=\int_{A} C(x, a) \varphi(\mathrm{d} a \mid x) \text { and } Q_{\varphi}(\cdot \mid x):=\int_{A} Q(\cdot \mid x, a) \varphi(\mathrm{d} a \mid x)
$$

for all $x \in \mathbb{X}$ and every stationary policy $\varphi$ in $\Phi$. 
Let $(\Omega, \mathcal{F})$ be the (canonical) measurable space consisting of the sample space

$$
\Omega:=(\mathbb{X} \times \mathbb{A})^{\infty}
$$

and its product $\sigma$-algebra $\mathcal{F}$. Then, for each policy $\pi$ and 'initial state' $x \in \mathbb{X}$, a stochastic process $\left\{\left(x_{n}, a_{n}\right)\right\}$ and a probability measure $\mathrm{P}_{x}^{\pi}$ are defined on $(\Omega, \mathcal{F})$ in a canonical way, where $x_{n}$ and $a_{n}$ represent the state and control at time $n, n=0,1, \ldots$ The expectation operator with respect to $\mathrm{P}_{x}^{\pi}$ is denoted by $\mathrm{E}_{x}^{\pi}$.

Let $W: \mathbb{X} \rightarrow[\theta, \infty)$ be a measurable function that will be referred to as a weight function, where $\theta>0$. We denote by $B_{W}(\mathbb{X})$ the normed linear space of measurable functions $u$ on $\mathbb{X}$ with finite $W$-norm $\|u\|_{W}$, which is defined as

$$
\|u\|_{W}:=\frac{\sup _{x \in \mathbb{X}}|u(x)|}{W(x)} .
$$

In this case we say that $u$ is $W$ bounded. Note that $B_{W}(\mathbb{X})$ is a Banach space.

Let $\mu(\cdot)$ be a measure on $\mathbb{X}$. We write

$$
\mu(u):=\int_{\mathbb{X}} u(y) \mu(\mathrm{d} y)
$$

whenever the integral is well defined.

Definition 2.1. Let

$$
S_{n}(\varphi, x):=\sum_{k=0}^{n-1} C_{\varphi}\left(x_{k}\right) \quad \text { and } \quad J_{n}(\varphi, x):=\mathrm{E}_{x}^{\varphi}\left[S_{n}(\varphi, x)\right]
$$

be the total pathwise $n$-stage cost and the total expected $n$-stage cost, respectively, when using the stationary policy $\varphi$, given the initial state $x_{0}=x$.

\section{Assumptions and main result}

In this section we introduce conditions to obtain asymptotic normality.

We will first introduce two sets of hypotheses. The first one, Assumption 3.1 below, uses a weight function $W$ to impose a growth condition on the cost function. The second one, Assumption 3.2, imposes in particular a Lyapunov-like condition. These assumptions will ensure the existence of solutions to the Poisson equation (PE) in (3.4) below (see Lemma 3.1).

Assumption 3.2 has previously been used for MCPs on Borel spaces (see, for instance, [1], [2], [7], and [10]), but it was always combined with additional conditions that imply $W$-geometric ergodicity. The approach in this paper is quite different, because we do not require $W$-geometric ergodicity.

Assumption 3.1. There exists a constant $K>0$ and a measurable weight function $W(\cdot)$ on $\mathbb{X}$ such that

(a) $W$ is bounded below by a constant $\theta>0$;

(b) $|C(x, a)| \leq K W(x)$ for all $(x, a) \in \mathbb{K}$.

We now state our second main assumption. 
Assumption 3.2. There exist a nontrivial finite measure $v(\cdot)$ on $\mathbb{X}$, a nonnegative measurable function $l(\cdot, \cdot)$ on $\mathbb{K}$, and a positive constant $\lambda<1$ such that

(a) $v(W)<\infty$;

(b) $Q(\cdot \mid x, a) \geq l(x, a) v(\cdot)$ for each $(x, a) \in \mathbb{K}$;

(c) $\int_{\mathbf{X}} W(y) Q(\mathrm{~d} y \mid x, a) \leq \lambda W(x)+l(x, a) v(W)$ for each $(x, a) \in \mathbb{K}$.

Remark 3.1. Assumption 3.1(a) and iterations of the inequality in Assumption 3.2(c) yield, for every $x \in \mathbb{X}, \pi \in \Pi$, and $n=0,1, \ldots$,

$$
\theta \leq \mathrm{E}_{x}^{\pi} W\left(x_{n}\right) \leq \lambda^{n} W(x)+\frac{v(W)}{(1-\lambda) v(\mathbb{X})} .
$$

This fact and (2.2) show that, for every $u \in B_{W}(\mathbb{X})$ and $p>0$,

$$
\lim _{n \rightarrow \infty} \frac{1}{n^{p}} \mathrm{E}_{x}^{\pi}\left|u\left(x_{n}\right)\right|=0 .
$$

In addition to Assumptions 3.1 and 3.2, we next impose other conditions on the control model. Several versions of these conditions have appeared in the literature (see, for instance, [3], [6], [7], [10], [21], and [22]), but the main ideas go back to [1] and [2].

Assumption 3.3. For each $x \in \mathbb{X}$,

(a) $A(x)$ is a (nonempty) compact subset of $\mathbb{A}$;

(b) $C(x, \cdot)$ is upper semicontinuous on $A(x)$;

(c) $Q(\cdot \mid x, \cdot)$ is strongly continuous on $A(x)$, that is, the mapping

$$
a \rightarrow \int_{\mathbb{X}} u(y) Q(\mathrm{~d} y \mid x, a)
$$

is continuous on $A(x)$ for each bounded measurable function $u$ on $\mathbb{X}$;

(d) the mapping $a \rightarrow \int_{\mathbb{X}} W(y) Q(\mathrm{~d} y \mid x, a)$ is continuous on $A(x)$, with $W$ as in Assumption 3.1;

(e) $l(x, \cdot)$ is continuous on $A(x)$, with $l(\cdot, \cdot)$ as in Assumption 3.2.

Suppose that Assumptions 3.1, 3.2, and 3.3 hold, and, in addition, that we replace Assumption 3.1(b) with a second-order condition, say $C^{2}(x, a) \leq K W(x)$. Then we can ensure the existence of control policies that maximize the long-run pathwise average cost in Section 1. (See, for instance, [6], [17], [20], and [23].) To obtain asymptotic normality, however, we need to strengthen the growth condition on the cost with the following fourth-order condition.

Assumption 3.4. There exists a positive constant $r_{2}$ such that

$$
C^{4}(x, a) \leq r_{2} W(x) \quad \text { for all }(x, a) \in \mathbb{K} .
$$

We now state some facts from [17, Theorem 2.1.4 and Lemma 4.2.2] which we need to present our main result. 
Lemma 3.1. (a) Under Assumptions 3.1 and 3.2, the following facts hold for each $\varphi \in \Phi$.

(i) The Markov chain defined by $Q_{\varphi}(\cdot \mid \cdot)$ is v-irreducible and positive Harris recurrent; hence, it admits a unique invariant probability measure, say $\mu_{\varphi}$.

(ii) $\mu_{\varphi}(W)<\infty$; thus, we have

$$
\rho_{\varphi}:=\mu_{\varphi}\left(C_{\varphi}\right)<\infty .
$$

(iii) There exists a function $h_{\varphi}^{*}$ in $B_{W}(\mathbb{X})$ such that the pair $\left(\rho_{\varphi}, h_{\varphi}^{*}\right)$ satisfies the $P E$

$$
h_{\varphi}^{*}(x)=C_{\varphi}(x)-\rho_{\varphi}+\int_{\mathbb{X}} h_{\varphi}^{*}(y) Q_{\varphi}(\mathrm{d} y \mid x) \text { for all } x \in \mathbb{X} .
$$

(b) Suppose that Assumptions 3.1, 3.2, 3.3, and 3.4 hold. Let $\varphi \in \Phi$ be arbitrary, and let $h_{\varphi}^{*}$ be as in (a)(iii). We define the function

$$
\Psi_{\varphi}(x):=\int_{\mathbb{X}} h_{\varphi}^{* 2}(y) Q_{\varphi}(\mathrm{d} y \mid x)-\left(\int_{\mathbb{X}} h_{\varphi}^{*}(y) Q_{\varphi}(\mathrm{d} y \mid x)\right)^{2}
$$

for each $x \in \mathbb{X}$. Then the functions $h_{\varphi}^{* 2}$ and $\Psi_{\varphi}$ belong to $B_{W}(\mathbb{X})$, and so $\sigma_{\varphi}^{2}:=\mu_{\varphi}\left(\Psi_{\varphi}\right)$ is finite.

Proof. Part (a) is an adaptation to our present context of Theorem 2.1.4 of [17]. On the other hand, under Assumptions 3.1(a) and 3.4, we have in particular that the cost function $C$ is $W^{1 / 2}$ bounded. It follows that Assumptions 3.1 and 3.2 are satisfied when we replace $W$ with $W^{1 / 2}$. Therefore, part (b) follows from (a).

We can now state our main result, which is proved in Section 4.

Theorem 3.1. Suppose that Assumptions 3.1, 3.2, 3.3, and 3.4 hold. Let $\varphi \in \Phi$ be a randomized stationary policy. Then, for every initial state $x \in \mathbb{X}$,

$$
\frac{S_{n}(\varphi, x)-n \rho_{\varphi}}{\sqrt{n}}
$$

has asymptotically a normal distribution $N\left(0, \sigma_{\varphi}^{2}\right)$ as $n \rightarrow \infty$, with $S_{n}(\varphi, x)$ as in Definition 2.1, and $\rho_{\varphi}$ and $\sigma_{\varphi}^{2}$ as defined in Lemma 3.1(a) and $(b)$, respectively.

Remark 3.2. Taking the action space $\mathbb{A}$ as a single-point set (or singleton), Theorem 3.1 becomes a central limit theorem for (noncontrolled) Markov chains.

\section{Proof of Theorem 3.1}

In this section we suppose that Assumptions 3.1,3.2, 3.3, and 3.4 hold. We need the following definition.

Definition 4.1. Let $J_{n}(\varphi, x)$ and $S_{n}(\varphi, x)$ be as in Definition 2.1. For every $\varphi \in \Phi$ and initial state $x$, we define the limiting average variance

$$
V(\varphi, x):=\limsup _{n \rightarrow \infty} \frac{1}{n} \operatorname{var}\left[S_{n}(\varphi, x)\right],
$$

where (by the definition of the variance of a random variable)

$$
\operatorname{var}\left[S_{n}(\varphi, x)\right]=\mathrm{E}_{x}^{\varphi}\left[S_{n}(\varphi, x)-J_{n}(\varphi, x)\right]^{2} .
$$


The following lemma is crucial to prove Theorem 3.1.

Lemma 4.1. Suppose that Assumptions 3.1, 3.2, 3.3, and 3.4 hold. Let $\varphi \in \Phi$ be arbitrary, and let $h_{\varphi}^{*}$ and $\Psi_{\varphi}$ be as in Lemma 3.1. Then the limiting average variance satisfies

$$
V(\varphi, x)=\lim _{n \rightarrow \infty} \frac{1}{n} \mathrm{E}_{x}^{\varphi} \sum_{k=0}^{n-1} \Psi_{\varphi}\left(x_{k}\right)=\sigma_{\varphi}^{2} \quad \text { for all } x \in \mathbb{X},
$$

with $\sigma_{\varphi}^{2}=\mu_{\varphi}\left(\Psi_{\varphi}\right)$ as in Lemma 3.1(b).

Proof. The following proof is taken from [4, Theorem 11.2.4] adapted to our present context.

From (3.4), an iteration procedure yields

$$
J_{n}(\varphi, x)=\mathrm{E}_{x}^{\varphi}\left[\sum_{k=0}^{n-1} C_{\varphi}\left(x_{k}\right)\right]=n \rho_{\varphi}+h_{\varphi}^{*}(x)-\mathrm{E}_{x}^{\varphi} h_{\varphi}^{*}\left(x_{n}\right) .
$$

Hence,

$$
S_{n}(\varphi, x)-J_{n}(\varphi, x)=\left[S_{n}(\varphi, x)-n \rho_{\varphi}\right]-\left[h_{\varphi}^{*}(x)-\mathrm{E}_{x}^{\varphi} h_{\varphi}^{*}\left(x_{n}\right)\right]
$$

and

$$
\operatorname{var}\left[S_{n}(\varphi, x)\right]=\mathrm{E}_{x}^{\varphi}\left[S_{n}(\varphi, x)-n \rho_{\varphi}\right]^{2}-\mathrm{E}_{x}^{\varphi}\left[h_{\varphi}^{*}(x)-\mathrm{E}_{x}^{\varphi} h_{\varphi}^{*}\left(x_{n}\right)\right]^{2} .
$$

Thus,

$$
\operatorname{var}\left[S_{n}(\varphi, x)\right]=\mathrm{E}_{x}^{\varphi}\left[S_{n}(\varphi, x)-n \rho_{\varphi}\right]^{2}+o_{1}(x, n)
$$

with

$$
o_{1}(x, n):=-\mathrm{E}_{x}^{\varphi}\left[h_{\varphi}^{*}(x)-\mathrm{E}_{x}^{\varphi} h_{\varphi}^{*}\left(x_{n}\right)\right]^{2} .
$$

We claim that

$$
\frac{o_{1}(x, n)}{n} \rightarrow 0 \text { for all } x \in \mathbb{X}
$$

as $n \rightarrow \infty$. Indeed,

$$
\left|o_{1}(x, n)\right| \leq 2\left[h_{\varphi}^{* 2}(x)+\mathrm{E}_{x}^{\varphi} h_{\varphi}^{* 2}\left(x_{n}\right)\right] .
$$

Hence, since $h_{\varphi}^{* 2} \in B_{W}(\mathbb{X})$ and by (3.2) in Remark 3.1, limit (4.4) holds.

On the other hand, define

$$
Y_{k}:=h_{\varphi}^{*}\left(x_{k}\right)-\mathrm{E}_{x}^{\varphi}\left[h_{\varphi}^{*}\left(x_{k}\right) \mid x_{k-1}\right], \quad k=1,2, \ldots
$$

From (4.5) we obtain

$$
Y_{k}^{2}=h_{\varphi}^{* 2}\left(x_{k}\right)-2 h_{\varphi}^{*}\left(x_{k}\right) \mathrm{E}_{x}^{\varphi}\left[h_{\varphi}^{*}\left(x_{k}\right) \mid x_{k-1}\right]+\left(\mathrm{E}_{x}^{\varphi}\left[h_{\varphi}^{*}\left(x_{k}\right) \mid x_{k-1}\right]\right)^{2} .
$$

Therefore,

$$
\begin{aligned}
\mathrm{E}_{x}^{\varphi}\left(Y_{k}^{2}\right) & =\mathrm{E}_{x}^{\varphi} h_{\varphi}^{* 2}\left(x_{k}\right)-\mathrm{E}_{x}^{\varphi}\left(\mathrm{E}_{x}^{\varphi}\left[h_{\varphi}^{*}\left(x_{k}\right) \mid x_{k-1}\right]\right)^{2} \\
& =\mathrm{E}_{x}^{\varphi} \mathrm{E}_{x}^{\varphi}\left[h_{\varphi}^{* 2}\left(x_{k}\right) \mid x_{k-1}\right]-\mathrm{E}_{x}^{\varphi}\left(\mathrm{E}_{x}^{\varphi}\left[h_{\varphi}^{*}\left(x_{k}\right) \mid x_{k-1}\right]\right)^{2} \\
& =\mathrm{E}_{x}^{\varphi}\left[\int_{\mathbb{X}} h_{\varphi}^{* 2}(y) Q_{\varphi}\left(\mathrm{d} y \mid x_{k-1}\right)-\left(\int_{\mathbb{X}} h_{\varphi}^{*}(y) Q_{\varphi}\left(\mathrm{d} y \mid x_{k-1}\right)\right)^{2}\right] \\
& =\mathrm{E}_{x}^{\varphi}\left[\Psi_{\varphi}\left(x_{k-1}\right)\right],
\end{aligned}
$$


that is,

$$
\mathrm{E}_{x}^{\varphi}\left(Y_{k}^{2}\right)=\mathrm{E}_{x}^{\varphi}\left[\Psi_{\varphi}\left(x_{k-1}\right)\right]
$$

Moreover, we have

$$
\operatorname{var}\left[S_{n}(\varphi, x)\right]=\mathrm{E}_{x}^{\varphi}\left[\sum_{k=1}^{n-1} Y_{k}^{2}\right]+o_{2}(x, n)
$$

with

$$
\frac{o_{2}(x, n)}{n} \rightarrow 0 \quad \text { when } n \rightarrow \infty
$$

To prove (4.7)-(4.8), note that the random variables $Y_{t}$ satisfy

$$
\mathrm{E}_{x}^{\varphi}\left[Y_{t} Y_{s}\right]=0 \quad \text { for all } 1 \leq t<s .
$$

We define the $\sigma$-algebras

$$
\mathscr{F}_{t}:=\sigma\left\{x_{0}, \ldots, x_{t}\right\} \quad \text { for } t=0,1, \ldots
$$

Then

$$
\mathrm{E}_{x}^{\varphi}\left[Y_{s} \mid \mathcal{F}_{s-1}\right]=\mathrm{E}_{x}^{\varphi}\left[h_{\varphi}^{*}\left(x_{s}\right) \mid x_{s-1}\right]-\mathrm{E}_{x}^{\varphi}\left[h_{\varphi}^{*}\left(x_{s}\right) \mid x_{s-1}\right]=0 \quad \text { for all } s,
$$

which implies that

$$
\mathrm{E}_{x}^{\varphi}\left[Y_{t} Y_{s}\right]=\mathrm{E}_{x}^{\varphi}\left[\mathrm{E}_{x}^{\varphi}\left[Y_{t} Y_{s} \mid \mathcal{F}_{s-1}\right]\right]=\mathrm{E}_{x}^{\varphi}\left[Y_{t} \mathrm{E}_{x}^{\varphi}\left[Y_{s} \mid \mathcal{F}_{s-1}\right]\right]=0 .
$$

On the other hand, since $h_{\varphi}^{*}$ satisfies the Poisson equation (3.4), we also have

$$
C_{\varphi}(x)-\rho_{\varphi}=h_{\varphi}^{*}(x)-\int_{\mathbb{X}} h_{\varphi}^{*}(y) Q_{\varphi}(\mathrm{d} y \mid x) \quad \text { for all } x \in \mathbb{X} .
$$

Consequently, by (4.5),

$$
\begin{aligned}
C_{\varphi}\left(x_{k}\right)-\rho_{\varphi} & =h_{\varphi}^{*}\left(x_{k}\right)-\int_{\mathbb{X}} h_{\varphi}^{*}(y) Q_{\varphi}\left(\mathrm{d} y \mid x_{k}\right) \\
& =Y_{k}+\int_{\mathbb{X}} h_{\varphi}^{*}(y) Q_{\varphi}\left(\mathrm{d} y \mid x_{k-1}\right)-\int_{\mathbb{X}} h_{\varphi}^{*}(y) Q_{\varphi}\left(\mathrm{d} y \mid x_{k}\right) \\
& =Y_{k}-\left[P h_{\varphi}^{*}\left(x_{k}\right)-P h_{\varphi}^{*}\left(x_{k-1}\right)\right] \quad \text { for all } k=1,2 \ldots,
\end{aligned}
$$

with

$$
P u(x):=\int_{\mathbb{X}} u(y) Q_{\varphi}(\mathrm{d} y \mid x) \text { for all } x \in \mathbb{X} \text { and } u \in B_{W}(\mathbb{X}) .
$$

From (4.10) we obtain

$$
S_{n}(\varphi, x)-n \rho_{\varphi}=M_{n-1}+\left[h_{\varphi}^{*}(x)-P h_{\varphi}^{*}\left(x_{n-1}\right)\right] \text { for all } n \geq 2
$$

with

$$
M_{n}:=\sum_{k=1}^{n} Y_{k} \quad \text { for all } n \geq 1 .
$$

Observe that (4.9) implies that

$$
\mathrm{E}_{x}^{\varphi}\left[M_{n}^{2}\right]=\mathrm{E}_{x}^{\varphi}\left[\sum_{k=1}^{n} Y_{k}^{2}\right]
$$


By (4.11),

$$
\begin{aligned}
\mathrm{E}_{x}^{\varphi}\left[S_{n}(\varphi, x)-n \rho_{\varphi}\right]^{2}= & \mathrm{E}_{x}^{\varphi}\left[M_{n-1}^{2}\right]+2 \mathrm{E}_{x}^{\varphi}\left[\left(h_{\varphi}^{*}(x)-P h_{\varphi}^{*}\left(x_{n-1}\right)\right) M_{n-1}\right] \\
& +\mathrm{E}_{x}^{\varphi}\left[h_{\varphi}^{*}(x)-P h_{\varphi}^{*}\left(x_{n-1}\right)\right]^{2} .
\end{aligned}
$$

Substituting this relation into (4.2), together with (4.12), we obtain (4.7) as we wanted to prove, with

$$
o_{2}(x, n):=o_{1}(x, n)+A(x, n)+2 B(x, n),
$$

where $o_{1}(x, n)$ is as in (4.3), and

$$
\begin{aligned}
& A(x, n):=\mathrm{E}_{x}^{\varphi}\left[h_{\varphi}^{*}(x)-P h_{\varphi}^{*}\left(x_{n-1}\right)\right]^{2}, \\
& B(x, n):=\mathrm{E}_{x}^{\varphi}\left[\left(h_{\varphi}^{*}(x)-P h_{\varphi}^{*}\left(x_{n-1}\right)\right) M_{n-1}\right] .
\end{aligned}
$$

To complete the proof of (4.7)-(4.8), we only need to show that, for every initial state $x$, as $n \rightarrow \infty$,

$$
\frac{A(x, n)}{n} \rightarrow 0
$$

and

$$
\frac{B(x, n)}{n} \rightarrow 0
$$

To prove (4.13), it suffices to note that

$$
A(x, n) \leq 2\left[h_{\varphi}^{* 2}(x)+\mathrm{E}_{x}^{\varphi} h_{\varphi}^{* 2}\left(x_{n}\right)\right] .
$$

This yields (4.13).

Now, to obtain (4.14), we use the Cauchy-Schwartz inequality to see that

$$
B(x, n)^{2} \leq \mathrm{E}_{x}^{\varphi}\left[h_{\varphi}^{*}(x)-P h_{\varphi}^{*}\left(x_{n-1}\right)\right]^{2} \mathrm{E}_{x}^{\varphi}\left[M_{n}^{2}\right]=A(x, n) \mathrm{E}_{x}^{\varphi}\left[\sum_{k=1}^{n} Y_{k}^{2}\right] .
$$

Hence, by (4.6),

$$
\frac{B(x, n)}{n} \leq \sqrt{\frac{A(x, n)}{n}} \sqrt{\frac{1}{n} \sum_{k=0}^{n-1} \mathrm{E}_{x}^{\varphi} \Psi_{\varphi}\left(x_{k}\right)}
$$

This inequality implies the limit in (4.14). This completes the proof of (4.7)-(4.8).

Combining (4.6), (4.7), and (4.8), we obtain

$$
\begin{aligned}
\operatorname{var}\left[S_{n}(\varphi, x)\right] & =\mathrm{E}_{x}^{\varphi}\left[\sum_{k=1}^{n-1} Y_{k}^{2}\right]+o_{2}(x, n) \\
& =\mathrm{E}_{x}^{\varphi}\left[\sum_{k=1}^{n} Y_{k}^{2}\right]+o_{2}(x, n)-\mathrm{E}_{x}^{\varphi} Y_{n}^{2} \\
& =\sum_{k=0}^{n-1} \mathrm{E}_{x}^{\varphi}\left[\Psi_{\varphi}\left(x_{k}\right)\right]+o_{2}(x, n)-\mathrm{E}_{x}^{\varphi} \Psi_{\varphi}\left(x_{n-1}\right) .
\end{aligned}
$$


Hence,

$$
V(\varphi, x)=\limsup _{n \rightarrow \infty} \frac{1}{n} \operatorname{var}\left[S_{n}(\varphi, x)\right]=\lim _{n \rightarrow \infty} \frac{1}{n} \sum_{k=0}^{n-1} \mathrm{E}_{x}^{\varphi}\left[\Psi_{\varphi}\left(x_{k}\right)\right]=\sigma_{\varphi}^{2},
$$

which proves (4.1).

Lemma 4.2. Under Assumptions 3.1, 3.2, 3.3, and 3.4, the following statements, for each $\varphi \in \Phi$, hold.

(a) The function $h_{\varphi}^{*}$ in Lemma 3.1(a) is $W^{1 / 4}$ bounded.

(b) The function $\Psi_{\varphi}$ in Lemma 3.1(b) is $W^{1 / 2}$ bounded. Moreover, there exists a $W^{1 / 2}$ bounded function $\widehat{h}_{\varphi}^{*}$ such that

$$
\widehat{h}_{\varphi}^{*}(x)=\Psi_{\varphi}(x)-\sigma_{\varphi}^{2}+\int_{\mathbb{X}} \widehat{h}_{\varphi}^{*}(y) Q_{\varphi}(\mathrm{d} y \mid x) \text { for all } x \in \mathbb{X},
$$

with $\sigma_{\varphi}^{2}$ as in (4.1).

Proof. (a) This part follows from Lemma 3.1 if we prove that Assumptions 3.1 and 3.2 are satisfied when we replace $W$ by $W^{1 / 4}$.

Indeed, under Assumption 3.4, Assumptions 3.1 and 3.2(a)-(b) obviously hold when we replace $W$ by $W^{1 / 4}$. It only remains to prove that Assumption 3.2(c) holds. To this end, define

$$
\widehat{Q}(B \mid x, a):=Q(B \mid x, a)-v(B) l(x, a)
$$

for each $B \in \mathcal{B}(\mathbb{X})$ and $(x, a) \in \mathbb{K}$. Under Assumption 3.2(b), $\widehat{Q}$ is a nonnegative kernel on $\mathbb{X}$ given $\mathbb{K}$. By the Cauchy-Schwartz inequality,

$$
\int_{\mathbb{X}} \sqrt{W(y)} \widehat{Q}(\mathrm{~d} y \mid x, a) \leq \sqrt{\int_{\mathbb{X}} W(y) \widehat{Q}(\mathrm{~d} y \mid x, a)} \sqrt{\widehat{Q}(\mathbb{X} \mid x, a)} \leq \sqrt{\lambda} \sqrt{W(x)}
$$

because $0 \leq \widehat{Q}(\mathbb{X} \mid x, a) \leq 1$. Hence, by the Cauchy-Schwartz inequality again, and from (4.17), we obtain

$$
\int_{\mathbb{X}} W(y)^{1 / 4} \widehat{Q}(\mathrm{~d} y \mid x, a) \leq \sqrt{\int_{\mathbb{X}} \sqrt{W(y)} \widehat{Q}(\mathrm{~d} y \mid x, a)} \sqrt{\widehat{Q}(\mathbb{X} \mid x, a)} \leq \lambda^{1 / 4} W(x)^{1 / 4} .
$$

From the definition of the kernel $\widehat{Q}$ in (4.16), we obtain

$$
\int_{\mathbb{X}} W(y)^{1 / 4} Q(\mathrm{~d} y \mid x, a) \leq \lambda^{1 / 4} W(x)^{1 / 4}+l(x, a) v\left(W^{1 / 4}\right) \quad \text { for all }(x, a) \in \mathbb{K} .
$$

This implies that Assumption 3.2(c) holds when we replace $W$ by $W^{1 / 4}$.

(b) Consider the cost function $C(x, a):=\Psi_{\varphi}(x)$ for all $(x, a) \in \mathbb{K}$. From part (a), $\Psi_{\varphi}$ is $W^{1 / 2}$ bounded. Applying Lemma 3.1 to this particular cost function we obtain the desired result.

We are finally ready to prove Theorem 3.1.

Proof of Theorem 3.1. From Lemmas 3.1(a)(iii) and 4.2(i), there exists a $W^{1 / 4}$-bounded function $h_{\varphi}^{*}$ satisfying (3.4). Similarly, from Lemma 4.2(ii), there exists a $W^{1 / 2}$-bounded function $\widehat{h}_{\varphi}^{*}$ satisfying (4.15). Let $h_{1}:=h_{\varphi}^{*}$ and $h_{2}=\widehat{h}_{\varphi}^{*}$. Thus, $h_{1}$ is $W^{1 / 4}$ bounded and $h_{2}$ is $W^{1 / 2}$ bounded. 
We also define

$$
\tau_{1}(x):=\int_{\mathbb{X}} h_{1}(y) Q_{\varphi}(\mathrm{d} y \mid x)-h_{1}(x)+C_{\varphi}(x)-\rho_{\varphi}
$$

and

$$
\tau_{2}(x):=\int_{\mathbb{X}} h_{2}(y) Q_{\varphi}(\mathrm{d} y \mid x)-h_{2}(x)+\Psi_{\varphi}(x)-\sigma_{\varphi}^{2}
$$

for all $x \in \mathbb{X}$.

For $l=1,2$ and $x \in \mathbb{X}$, let

$$
\psi_{l}(x):=\int_{\mathbb{X}} h_{l}(y) Q_{\varphi}(\mathrm{d} y \mid x)-h_{l}(x) .
$$

Observe that

$$
\tau_{1}(x)=\psi_{1}(x)+C_{\varphi}(x)-\rho_{\varphi}
$$

and

$$
\tau_{2}(x)=\psi_{2}(x)+\Psi_{\varphi}(x)-\sigma_{\varphi}^{2}
$$

for all $(x, a) \in \mathbb{K}$.

Consider the characteristic functions $\mathrm{E}_{x}^{\varphi} \chi_{n}(u)$ with

$$
\chi_{n}(u):=\exp \left\{\mathrm{i} u\left(S_{n}(\varphi, x)-n \rho_{\varphi}\right)\right\} \quad \text { for } n=1,2, \ldots, u \in \mathbb{R},
$$

and $\chi_{0}(u):=1$. Let

$$
\begin{aligned}
& e_{1}(z):=\exp \{\mathrm{i} z\}-\mathrm{i} z-1, \\
& e_{2}(z):=\exp \{\mathrm{i} z\}+\frac{z^{2}}{2}-\mathrm{i} z-1,
\end{aligned}
$$

for all $z$ in $\mathbb{R}$.

To prove the theorem, we have to verify that

$$
\lim _{n \rightarrow \infty} \mathrm{E}_{x}^{\varphi} \chi_{n}\left(\frac{u}{\sqrt{n}}\right)=\exp \left\{-\frac{1}{2} \sigma_{\varphi}^{2} u^{2}\right\} \quad \text { for all } u \in \mathbb{R} .
$$

To this end, fix an arbitrary $u \in \mathbb{R}$. First note that $\psi_{l}\left(x_{m}\right)$ for $l=1,2$ is the conditional expectation of $h_{l}\left(x_{m+1}\right)-h_{l}\left(x_{m}\right)$ given $x_{m}$, that is,

$$
\psi_{l}\left(x_{m}\right)=\mathrm{E}_{x}^{\varphi}\left[h_{l}\left(x_{m+1}\right)-h_{l}\left(x_{m}\right) \mid x_{m}\right] .
$$

This yields, for $l=1,2$, with $\chi_{m}:=\chi_{m}(u)$, the equations

$$
0=\mathrm{i} u \mathrm{E}_{x}^{\varphi}\left[\sum_{m=0}^{n-1} \chi_{m} \psi_{1}\left(x_{m}\right)-\sum_{m=0}^{n-1} \chi_{m}\left(h_{1}\left(x_{m+1}\right)-h_{1}\left(x_{m}\right)\right)\right]
$$

and

$$
0=\frac{u^{2}}{2} \mathrm{E}_{x}^{\varphi}\left[\sum_{m=0}^{n-1} \chi_{m}\left(h_{2}\left(x_{m+1}\right)-h_{2}\left(x_{m}\right)\right)-\sum_{m=0}^{n-1} \chi_{m} \psi_{2}\left(x_{m}\right)\right]
$$


To simplify the notation, let $e_{1}:=e_{1}\left(u\left(C_{\varphi}\left(x_{m}\right)-\rho_{\varphi}\right)\right)$ and $e_{2}:=e_{2}\left(u\left(C_{\varphi}\left(x_{m}\right)-\rho_{\varphi}\right)\right)$. Moreover, note that

$$
\chi_{m+1}-\chi_{m}=\left[\exp \left\{\mathrm{i} u\left(C_{\varphi}\left(x_{m}\right)-\rho_{\varphi}\right)\right\}-1\right] \chi_{m} .
$$

From (4.20), (4.21), and (4.25), we have

$$
\begin{aligned}
\mathrm{E}_{x}^{\varphi} \chi_{n}-1 & =\mathrm{E}_{x}^{\varphi} \sum_{m=0}^{n-1}\left(\chi_{m+1}-\chi_{m}\right) \\
& =\mathrm{E}_{x}^{\varphi} \sum_{m=0}^{n-1}\left[\mathrm{i} u\left(C_{\varphi}\left(x_{m}\right)-\rho_{\varphi}\right)-\frac{1}{2} u^{2}\left(C_{\varphi}\left(x_{m}\right)-\rho_{\varphi}\right)^{2}+e_{2}\right] \chi_{m}
\end{aligned}
$$

and

$$
\begin{aligned}
&-\mathrm{i} u \mathrm{E}_{x}^{\varphi} \sum_{m=0}^{n-1} \chi_{m}\left(h_{1}\left(x_{m+1}\right)-h_{1}\left(x_{m}\right)\right) \\
&=\mathrm{i} u \mathrm{E}_{x}^{\varphi}\left[h_{1}\left(x_{0}\right)-\chi_{n} h_{1}\left(x_{n}\right)+\sum_{m=0}^{n-1} h_{1}\left(x_{m+1}\right)\left(\chi_{m+1}-\chi_{m}\right)\right] \\
&=\mathrm{i} u \mathrm{E}_{x}^{\varphi}\left[h_{1}\left(x_{0}\right)-\chi_{n} h_{1}\left(x_{n}\right)+\sum_{m=0}^{n-1} h_{1}\left(x_{m+1}\right)\left(\mathrm{i} u\left(C_{\varphi}\left(x_{m}\right)-\rho_{\varphi}\right)+e_{1}\right) \chi_{m}\right] .
\end{aligned}
$$

Similarly,

$$
\begin{aligned}
\frac{u^{2}}{2} \mathrm{E}_{x}^{\varphi} & \sum_{m=0}^{n-1} \chi_{m}\left(h_{2}\left(x_{m+1}\right)-h_{2}\left(x_{m}\right)\right) \\
& =-\frac{u^{2}}{2} \mathrm{E}_{x}^{\varphi}\left[h_{2}\left(x_{0}\right)-\chi_{n} h_{2}\left(x_{n}\right)+\sum_{m=0}^{n-1} h_{2}\left(x_{m+1}\right)\left(\chi_{m+1}-\chi_{m}\right)\right] \\
& =-\frac{u^{2}}{2} \mathrm{E}_{x}^{\varphi}\left[h_{2}\left(x_{0}\right)-\chi_{n} h_{2}\left(x_{n}\right)+\sum_{m=0}^{n-1} h_{2}\left(x_{m+1}\right)\left(\exp \left\{\mathrm{i} u\left(C_{\varphi}\left(x_{m}\right)-\rho_{\varphi}\right)\right\}-1\right) \chi_{m}\right]
\end{aligned}
$$

Adding (4.23), (4.24), (4.26)-(4.28), and using (4.18),

$$
\begin{aligned}
\mathrm{E}_{x}^{\varphi} \chi_{n}-1 & \\
= & \mathrm{i} u \mathrm{E}_{x}^{\varphi}\left[h_{1}\left(x_{0}\right)-\chi_{n} h_{1}\left(x_{n}\right)+\sum_{m=0}^{n-1} \chi_{m} \tau_{1}\left(x_{m}\right)+\sum_{m=0}^{n-1} e_{1} h_{1}\left(x_{m+1}\right) \chi_{m}\right] \\
& -\frac{u^{2}}{2} \mathrm{E}_{x}^{\varphi} \sum_{m=0}^{n-1} \chi_{m}\left\{\psi_{2}\left(x_{m}\right)+2 h_{1}\left(x_{m+1}\right)\left(C_{\varphi}\left(x_{m}\right)-\rho_{\varphi}\right)+\left(C_{\varphi}\left(x_{m}\right)-\rho_{\varphi}\right)^{2}\right\} \\
& -\frac{u^{2}}{2} \mathrm{E}_{x}^{\varphi}\left[h_{2}\left(x_{0}\right)-\chi_{n} h_{2}\left(x_{n}\right)+\sum_{m=0}^{n-1} h_{2}\left(x_{m+1}\right)\left(\exp \left\{\mathrm{i} u\left(C_{\varphi}\left(x_{m}\right)-\rho_{\varphi}\right)\right\}-1\right) \chi_{m}\right] \\
& +\mathrm{E}_{x}^{\varphi} \sum_{m=0}^{n-1} e_{2} \chi_{m} .
\end{aligned}
$$




\section{Hence,}

$$
\begin{aligned}
\mathrm{E}_{x}^{\varphi} \chi_{n}-1= & \kappa^{\prime \prime}(n, u) \\
& -\frac{u^{2}}{2} \mathrm{E}_{x}^{\varphi} \sum_{m=0}^{n-1} \chi_{m}\left\{\psi_{2}\left(x_{m}\right)+2 h_{1}\left(x_{m+1}\right)\left(C_{\varphi}\left(x_{m}\right)-\rho_{\varphi}\right)+\left(C_{\varphi}\left(x_{m}\right)-\rho_{\varphi}\right)^{2}\right\}
\end{aligned}
$$

with

$$
\begin{aligned}
\kappa^{\prime \prime}(n, u):= & \mathrm{i} u \mathrm{E}_{x}^{\varphi}\left[h_{1}\left(x_{0}\right)-\chi_{n} h_{1}\left(x_{n}\right)+\sum_{m=0}^{n-1} \chi_{m} \tau_{1}\left(x_{m}\right)+\sum_{m=0}^{n-1} e_{1} h_{1}\left(x_{m+1}\right) \chi_{m}\right] \\
& -\frac{u^{2}}{2} \mathrm{E}_{x}^{\varphi}\left[h_{2}\left(x_{0}\right)-\chi_{n} h_{2}\left(x_{n}\right)+\sum_{m=0}^{n-1} h_{2}\left(x_{m+1}\right)\left(\exp \left\{\mathrm{i} u\left(C_{\varphi}\left(x_{m}\right)-\rho_{\varphi}\right)\right\}-1\right) \chi_{m}\right] \\
& +\mathrm{E}_{x}^{\varphi} \sum_{m=0}^{n-1} e_{2} \chi_{m} .
\end{aligned}
$$

From (3.5) we observe that

$$
\Psi_{\varphi}\left(x_{m}\right)=\mathrm{E}_{x}^{\varphi}\left[h_{1}^{2}\left(x_{m+1}\right) \mid x_{m}\right]-\left(\mathrm{E}_{x}^{\varphi}\left[h_{1}\left(x_{m+1}\right) \mid x_{m}\right]\right)^{2},
$$

and in view of (4.19), we can express (4.29) as

$$
\begin{aligned}
& \mathrm{E}_{x}^{\varphi} \chi_{n}-1 \\
& =\kappa^{\prime \prime}(n, u) \\
& \quad-\frac{u^{2}}{2} \mathrm{E}_{x}^{\varphi} \sum_{m=0}^{n-1} \chi_{m}\left\{\sigma_{\varphi}^{2}+\tau_{2}\left(x_{m}\right)-h_{1}^{2}\left(x_{m+1}\right)\left(\mathrm{E}_{x}^{\varphi}\left[h_{1}\left(x_{m+1}\right) \mid x_{m}\right]+C_{\varphi}\left(x_{m}\right)-\rho_{\varphi}\right)^{2}\right\} \\
& =\kappa^{\prime \prime}(n, u) \\
& \quad-\frac{u^{2}}{2} \mathrm{E}_{x}^{\varphi} \sum_{m=0}^{n-1} \chi_{m}\left\{\sigma_{\varphi}^{2}+\tau_{2}\left(x_{m}\right)-h_{1}^{2}\left(x_{m+1}\right)\right. \\
& \left.\quad+\left(\int_{\mathbb{X}} h_{1}(y) Q_{\varphi}\left(\mathrm{d} y \mid x_{m}\right)+C_{\varphi}\left(x_{m}\right)-\rho_{\varphi}\right)^{2}\right\} .
\end{aligned}
$$

Since $h_{1}=h_{\varphi}^{*}$ satisfies (3.4), we obtain

$$
h_{1}\left(x_{m}\right)=\int_{\mathbb{X}} h_{1}(y) Q_{\varphi}\left(\mathrm{d} y \mid x_{m}\right)+C_{\varphi}\left(x_{m}\right)-\rho_{\varphi} .
$$

Then, from (4.25), we have

$$
\begin{aligned}
\mathrm{E}_{x}^{\varphi} \chi_{n}-1= & \kappa^{\prime \prime}(n, u)-\frac{u^{2}}{2} \mathrm{E}_{x}^{\varphi} \sum_{m=0}^{n-1} \chi_{m}\left\{\sigma_{\varphi}^{2}+\tau_{2}\left(x_{m}\right)-h_{1}^{2}\left(x_{m+1}\right)+h_{1}^{2}\left(x_{m}\right)\right\} \\
= & \kappa^{\prime \prime}(n, u)-\frac{u^{2} \sigma_{\varphi}^{2}}{2} \sum_{m=0}^{n-1} \mathrm{E}_{x}^{\varphi} \chi_{m} \\
& -\frac{u^{2}}{2} \mathrm{E}_{x}^{\varphi}\left[h_{1}^{2}\left(x_{0}\right)-\chi_{n} h_{1}^{2}\left(x_{n}\right)+\sum_{m=0}^{n-1} \chi_{m} \tau_{2}\left(x_{m}\right)+\sum_{m=0}^{n-1} h_{1}^{2}\left(x_{m+1}\right)\left(\chi_{m+1}-\chi_{m}\right)\right]
\end{aligned}
$$




$$
\begin{aligned}
=\kappa^{\prime \prime}(n, u) & -\frac{u^{2} \sigma_{\varphi}^{2}}{2} \sum_{m=0}^{n-1} \mathrm{E}_{x}^{\varphi} \chi_{m} \\
-\frac{u^{2}}{2} \mathrm{E}_{x}^{\varphi}[ & h_{1}^{2}\left(x_{0}\right)-\chi_{n} h_{1}^{2}\left(x_{n}\right)+\sum_{m=0}^{n-1} \chi_{m} \tau_{2}\left(x_{m}\right) \\
& \left.+\sum_{m=0}^{n-1} h_{1}^{2}\left(x_{m+1}\right)\left(\exp \left\{\mathrm{i} u\left(C_{\varphi}\left(x_{m}\right)-\rho_{\varphi}\right)\right\}-1\right) \chi_{m}\right] .
\end{aligned}
$$

Hence,

$$
\mathrm{E}_{x}^{\varphi} \chi_{n}=1-\frac{u^{2} \sigma_{\varphi}^{2}}{2} \sum_{m=0}^{n-1} \mathrm{E}_{x}^{\varphi} \chi_{m}+\kappa^{\prime}(n, u)
$$

with

$$
\begin{aligned}
\kappa^{\prime}(n, u):=\kappa^{\prime \prime}(n, u)-\frac{u^{2}}{2} \mathrm{E}_{x}^{\varphi}[ & h_{1}^{2}\left(x_{0}\right)-\chi_{n} h_{1}^{2}\left(x_{n}\right)+\sum_{m=0}^{n-1} \chi_{m} \tau_{2}\left(x_{m}\right) \\
& \left.+\sum_{m=0}^{n-1} h_{1}^{2}\left(x_{m+1}\right)\left(\exp \left\{\mathrm{i} u\left(C_{\varphi}\left(x_{m}\right)-\rho_{\varphi}\right)\right\}-1\right) \chi_{m}\right]
\end{aligned}
$$

Let us rewrite (4.31) as

$$
\mathrm{E}_{x}^{\varphi} \chi_{n}=1+\left(\exp \left\{-\frac{u^{2} \sigma_{\varphi}^{2}}{2}\right\}-1\right) \sum_{m=0}^{n-1} \mathrm{E}_{x}^{\varphi} \chi_{m}+\kappa(n, u)
$$

with

$$
\kappa(n, u):=\kappa^{\prime}(n, u)+\left[1-\frac{u^{2} \sigma_{\varphi}^{2}}{2}-\exp \left\{-\frac{u^{2} \sigma_{\varphi}^{2}}{2}\right\}\right] \sum_{m=0}^{n-1} \mathrm{E}_{x}^{\varphi} \chi_{m} .
$$

Defining $\kappa(0, u):=0$, from (4.33), an induction argument gives

$$
\begin{aligned}
\mathrm{E}_{x}^{\varphi} \chi_{n}(u)= & \exp \left\{-\frac{n \sigma_{\varphi}^{2} u^{2}}{2}\right\} \\
& +\left[\exp \left\{-\frac{\sigma_{\varphi}^{2} u^{2}}{2}\right\}-1\right] \sum_{m=0}^{n-1} \exp \left\{-\frac{\sigma_{\varphi}^{2} u^{2}}{2}(n-1-m)\right\} \kappa(m, u)+\kappa(n, u) .
\end{aligned}
$$

Observe that the proof of limit (4.22) and, consequently, of Theorem 3.1 follows from (4.35) if we show that

$$
\max _{1 \leq m \leq n}\left|\kappa\left(m, \frac{u}{\sqrt{n}}\right)\right| \rightarrow 0 \quad \text { as } n \rightarrow \infty .
$$

This relation is obtained by an inspection of the different terms of $\kappa(m, u / \sqrt{n})$. We will do this in the following six steps.

Step 1. From the definition of $h_{1}=h_{\varphi}^{*}$ and $h_{2}=\widehat{h}_{\varphi}^{*}$ satisfying (3.4) and (4.15), we have $\tau_{1}\left(x_{m}\right)=0$ in (4.30) and $\tau_{2}\left(x_{m}\right)=0$ in (4.32) for $m=0,1, \ldots$ 
Step 2. By (3.1) we obtain

$$
\lim _{n \rightarrow \infty} \frac{1}{\sqrt{n}} \mathrm{E}_{x}^{\varphi} h\left(x_{n}\right)=0 \text { and } \lim _{n \rightarrow \infty} \frac{1}{n} \mathrm{E}_{x}^{\varphi} h\left(x_{n}\right)=0
$$

for every $h$ in $B_{W}(\mathbb{X})$. This limit appears in (4.30) and (4.32) when we replace $u$ by $u / \sqrt{n}$.

Step 3. In this part we prove the limit (see (4.30))

$$
\lim _{n \rightarrow \infty} \frac{1}{\sqrt{n}} \mathrm{E}_{x}^{\varphi} \sum_{m=0}^{n-1} e_{1} h_{1}\left(x_{m+1}\right) \chi_{m}=0 .
$$

Indeed, since $\left|e_{1}(z)\right| \leq z^{2} / 2$ for all $z$ in $\mathbb{R}$, we obtain

$$
\begin{aligned}
\left|\frac{1}{\sqrt{n}} \mathrm{E}_{x}^{\varphi} \sum_{m=0}^{n-1} e_{1} h_{1}\left(x_{m+1}\right) \chi_{m}\right| & \leq \frac{1}{2 \sqrt{n}} \mathrm{E}_{x}^{\varphi} \sum_{m=0}^{n-1} \frac{u^{2}}{n}\left|h_{1}\left(x_{m+1}\right)\right|\left(C_{\varphi}\left(x_{m}\right)-\rho_{\varphi}\right)^{2} \\
& =\frac{u^{2}}{2 n^{3 / 2}} \mathrm{E}_{x}^{\varphi} \sum_{m=0}^{n-1}\left|\int_{\mathbb{X}} h_{1}(y) Q_{\varphi}\left(\mathrm{d} y \mid x_{m}\right)\right|\left(C_{\varphi}\left(x_{m}\right)-\rho_{\varphi}\right)^{2}
\end{aligned}
$$

By Lemma 4.2(i), $h_{1}(\cdot)$ is $W^{1 / 4}$ bounded, in particular $h_{1}(\cdot)$ is $W^{1 / 2}$ bounded. Hence, the function $\int_{\mathbb{X}} h_{1}(y) Q_{\varphi}(\mathrm{d} y \mid \cdot)$ is $W^{1 / 2}$ bounded. On the other hand, by Assumption 3.4, $\left(C_{\varphi}(x)-\right.$ $\left.\rho_{\varphi}\right)^{2}$ is also $W^{1 / 2}$ bounded. Therefore,

$$
\left|\frac{1}{\sqrt{n}} \mathrm{E}_{x}^{\varphi} \sum_{m=0}^{n-1} e_{1} h_{1}\left(x_{m+1}\right) \chi_{m}\right| \leq \frac{\zeta u^{2}}{2 n^{3 / 2}} \mathrm{E}_{x}^{\varphi} \sum_{m=0}^{n-1} W\left(x_{m}\right),
$$

where $\zeta$ is a constant depending on $h_{1}$ and C. By Assumptions 3.1(a) and 3.2(c), and (3.1),

$$
\left|\frac{1}{\sqrt{n}} \mathrm{E}_{x}^{\varphi} \sum_{m=0}^{n-1} e_{1} h_{1}\left(x_{m+1}\right) \chi_{m}\right| \leq \frac{\zeta u^{2}}{2 n^{3 / 2}} n\left[1+\frac{v(W)}{(1-\lambda) \theta v(\mathbb{X})}\right] W(x),
$$

which converges to 0 as $n \rightarrow \infty$.

Step 4. We will next prove that

$$
\lim _{n \rightarrow \infty} \frac{1}{n} \mathrm{E}_{x}^{\varphi} \sum_{m=0}^{n-1} e_{2} \chi_{m}=0
$$

This limit appears in (4.30) when we replace $u$ by $u / \sqrt{n}$.

Observe that $\left|e_{2}(z)\right| \leq|z|^{3} / 6$ for all $z$ in $\mathbb{R}$. So, by Assumptions 3.1(a), 3.2(c), and 3.4, together with (3.1),

$$
\begin{aligned}
\left|\frac{1}{n} \mathrm{E}_{x}^{\varphi} \sum_{m=0}^{n-1} e_{2} \chi_{m}\right| & \leq \frac{|u|^{3}}{6 n^{5 / 2}} \mathrm{E}_{x}^{\varphi} \sum_{m=0}^{n-1}\left|C_{\varphi}\left(x_{m}\right)-\rho_{\varphi}\right|^{3} \\
& \leq \frac{k^{3}|u|^{3}}{6 n^{5 / 2}} \mathrm{E}_{x}^{\varphi} \sum_{m=0}^{n-1} W\left(x_{m}\right)^{3 / 4}
\end{aligned}
$$




$$
\begin{aligned}
& \leq \frac{k^{3}|u|^{3}}{6 n^{5 / 2} \theta^{1 / 4}} \mathrm{E}_{x}^{\varphi} \sum_{m=0}^{n-1} W\left(x_{m}\right) \\
& \leq \frac{k^{3}|u|^{3}}{6 n^{3 / 2}}\left(1+\frac{v(W)}{(1-\lambda) \theta v(\mathbb{X})}\right) W(x),
\end{aligned}
$$

which converges to 0 as $n \rightarrow \infty$, with $k$ a constant.

Step 5. Let $h$ be a $W^{1 / 2}$-bounded function on $\mathbb{X}$. Then

$$
\lim _{n \rightarrow \infty} \frac{1}{n} \mathrm{E}_{x}^{\varphi} \sum_{m=0}^{n-1} h\left(x_{m+1}\right)\left(\exp \left\{\mathrm{i} \frac{u}{\sqrt{n}}\left(C-\rho_{\varphi}\right)\right\}-1\right) \chi_{m}=0 .
$$

This limit appears in (4.30) and (4.32) when $u$ is replaced by $u / \sqrt{n}$.

From (4.20), $e_{1}(z)=\exp \{\mathrm{i} z\}-\mathrm{i} z-1$ and so

$$
\exp \left\{\mathrm{i} \frac{u}{\sqrt{n}}\left(C-\rho_{\varphi}\right)\right\}-1=\mathrm{i} \frac{u}{\sqrt{n}}\left(C-\rho_{\varphi}\right)+e_{1} \frac{u}{\sqrt{n}}\left(C-\rho_{\varphi}\right) .
$$

Hence,

$$
\begin{aligned}
& \mid \frac{1}{n} \mathrm{E}_{x}^{\varphi} \sum_{m=0}^{n-1} h\left(x_{m+1}\right)\left(\exp \left\{\mathrm{i} \frac{u}{\sqrt{n}}\left(C-\rho_{\varphi}\right)\right\}-1\right) \chi_{m} \mid \\
& \quad \leq \frac{|u|}{n^{3 / 2}} \mathrm{E}_{x}^{\varphi} \sum_{m=0}^{n-1}\left|h\left(x_{m+1}\right)\right|\left|\left(C_{\varphi}\left(x_{m}\right)-\rho_{\varphi}\right)\right|+\frac{1}{n} \mathrm{E}_{x}^{\varphi} \sum_{m=0}^{n-1}\left|h\left(x_{m+1}\right)\right|\left|e_{1}\right| .
\end{aligned}
$$

This gives the desired conclusion by similar arguments to those in step 3 .

Step 6. The absolute value of the expression within the brackets in (4.34) is majorized by $\sigma_{\varphi}^{4} u^{4} / 8$, and so the corresponding term in $\kappa(n, u / \sqrt{n})$ is majorized by $\sigma_{\varphi}^{4} u^{4} / 8 n^{2}$.

Steps 1-6 imply (4.36), and consequently prove Theorem 3.1 .

\section{Example: an LQ system}

In this section we consider a linear-quadratic (or LQ) system consisting of the linear system

$$
x_{t+1}=k_{1} x_{t}+k_{2} a_{t}+z_{t}, \quad t=0,1, \ldots,
$$

with state space $\mathbb{X}:=\mathbb{R}$, and positive coefficients $k_{1}$ and $k_{2}$. The control set is $A:=\mathbb{R}$, and the set of admisible controls in each state $x$ is the interval

$$
A(x):=\left[-\frac{k_{1}|x|}{k_{2}}, \frac{k_{1}|x|}{k_{2}}\right] .
$$

The disturbances $z_{t}$ are independent and identically distributed (i.i.d.) random variables with values in $Z:=\mathbb{R}$, zero mean, and finite variance, that is,

$$
\mathrm{E}\left[z_{t}\right]=0, \quad \sigma^{2}:=\mathrm{E}\left[z_{t}^{2}\right]<\infty .
$$

To complete the description of our control model, we introduce the quadratic cost-per-stage function

$$
C(x, a):=c_{1} x^{2}+c_{2} a^{2} \quad \text { for all }(x, a) \in \mathbb{K},
$$


with positive coefficients $c_{1}$ and $c_{2}$. We also define

$$
W(x):=\exp \{\gamma|x|\} \quad \text { for all } x \in \mathbb{X},
$$

with $\gamma \geq 4$. Clearly, Assumption 3.4 holds. Now let $\hat{s}>0$ be such that

$$
\gamma \hat{s}<\log \left(\frac{\gamma}{2}+1\right)
$$

which implies that

$$
\lambda:=\frac{2}{\gamma}(\exp \{\gamma \hat{s}\}-1)<1 .
$$

Throughout the rest of this section, we suppose that the following assumptions taken from [7, Section 5] hold.

Assumption 5.1. We have $0<k_{1}<\frac{1}{2}$.

Assumption 5.2. The i.i.d. disturbances $z_{t}$ have a common density $g$, which is a continuous bounded function supported on the interval $S:=[-\hat{s}, \hat{s}]$. Moreover, there exists a positive number $\varepsilon$ such that $g(s) \geq \varepsilon$ for all $s \in S$.

Assumptions 5.1 and 5.2 imply that Assumptions 3.1, 3.2, and 3.3 hold (see, for instance, [7, Propositions 6, 23, and 24]).

Now consider the stationary policy defined by the stochastic kernel:

$$
\varphi(\mathrm{d} a \mid x):= \begin{cases}\frac{k_{2}}{2 k_{1}|x|} \mathbf{1}_{A(x)}(a) \mathrm{d} a & \text { if } x \neq 0, \\ \delta_{0}(a) \mathrm{d} a & \text { if } x=0,\end{cases}
$$

where $\mathbf{1}$ is the usual indicator function. The corresponding cost function $C_{\varphi}$ can be obtained from (2.1). Indeed, a direct calculation gives, for every $x$,

$$
C_{\varphi}(x)=\widehat{c} x^{2} \quad \text { with } \quad \widehat{c}:=c_{1}+\frac{c_{2} k_{1}^{2}}{3 k_{2}^{2}}
$$

for all $x \in \mathbb{X}$. In this case, the pair $h_{\varphi}^{*}$ and $\rho_{\varphi}$ satisfying the PE (3.4) becomes

$$
\rho_{\varphi}=v_{0} \sigma^{2} \text { and } h_{\varphi}^{*}(x)=v_{0} x^{2},
$$

with $\sigma^{2}$ as in (5.1) and

$$
v_{0}=\frac{\widehat{c}}{1-4 / 3 k_{1}^{2}}
$$

Moreover, $\Psi_{\varphi}$ in (3.5) becomes

$$
\Psi_{\varphi}(x)=v_{0}^{2}\left(\frac{64}{45} k_{1}^{4} x^{4}+\frac{16}{3} \sigma^{2} k_{1}^{2} x^{2}+4 B k_{1} x+D-\sigma^{4}\right),
$$

with

$$
B:=\int z^{3} g(z) \mathrm{d} z, \quad \text { and } \quad D:=\int z^{4} g(z) \mathrm{d} z
$$


On the other hand, for every $t=1,2, \ldots$, we have

$$
\begin{gathered}
\mathrm{E}_{x}^{\varphi} x_{t+1}=k_{1} \mathrm{E}_{x}^{\varphi} x_{t}, \\
\mathrm{E}_{x}^{\varphi} x_{t+1}^{2}=\frac{4}{3} k_{1}^{2} \mathrm{E}_{x}^{\varphi} x_{t}^{2}+\sigma^{2},
\end{gathered}
$$

and

$$
\mathrm{E}_{x}^{\varphi} x_{t+1}^{4}=\frac{16}{5} k_{1}^{4} \mathrm{E}_{x}^{\varphi} x_{t}^{4}+8 \sigma^{2} k_{1}^{2} \mathrm{E}_{x}^{\varphi} x_{t}^{2}+4 B k_{1} \mathrm{E}_{x}^{\varphi} x_{t}+D .
$$

From (5.5) and (5.6)-(5.8), it follows that $\sigma_{\varphi}^{2}$, defined in Lemma 3.1(b), is given by

$$
\sigma_{\varphi}^{2}=\frac{v_{0}^{2}\left(45-80 k_{1}^{4}\right)}{9\left(5-16 k_{1}^{4}\right)}\left(D-\frac{3\left(448 k_{1}^{6}-144 k_{1}^{4}-300 k_{1}^{2}+45\right)}{\left(3-4 k_{1}^{2}\right)\left(45-80 k_{1}^{4}\right)} \sigma^{4}\right) .
$$

To conclude, by Theorem 3.1 and considering (5.3), it follows that, for every initial state $x \in \mathbb{X}$, as $n \rightarrow \infty$, the distribution of the cost

$$
\frac{\sum_{t=0}^{n-1} C_{\varphi}\left(x_{t}\right)-n v_{0} \sigma^{2}}{\sqrt{n}}
$$

has an asymptotic normal distribution $N\left(0, \sigma_{\varphi}^{2}\right)$ with $\sigma_{\varphi}^{2}$ as in (5.9).

Finally, by (5.2) and (5.4), it follows that, for every initial state $x$, as $n \rightarrow \infty$,

$$
\frac{\sum_{t=0}^{n-1} x_{t}^{2}-3 n \sigma^{2} /\left(3-4 k_{1}^{2}\right)}{\sqrt{n}}
$$

has an asymptotic normal distribution $N\left(0, s^{2}\right)$, where

$$
s^{2}=\frac{45-80 k_{1}^{4}}{\left(3-4 k_{1}^{2}\right)\left(5-16 k_{1}^{4}\right)}\left(D-\frac{3\left(448 k_{1}^{6}-144 k_{1}^{4}-300 k_{1}^{2}+45\right)}{\left(3-4 k_{1}^{2}\right)\left(45-80 k_{1}^{4}\right)} \sigma^{4}\right) .
$$

\section{References}

[1] Gordienko, E. and Hernández-Lerma, O. (1995). Average cost Markov control processes with weigthed norms: existence of canonical policies. Appl. Math. 23, 199-218.

[2] Gordienko, E. And Hernández-Lerma, O. (1995). Average cost Markov control processes with weigthed norms: value iteration. Appl. Math. 23, 219-237.

[3] Hernández-Lerma, O. And Lasserre, J. B. (1996). Discrete-Time Markov Control Processes. Springer, New York.

[4] Hernández-Lerma, O. and Lasserre, J. B. (1999). Further Topics on Discrete-time Markov Control Processes. Springer, New York.

[5] Hernández-Lerma, O. and Lasserre, J. B. (2001). Zero-sum stochastic games in Borel spaces: average payoff criteria. SIAM J. Control Optimization 39, 1520-1539.

[6] Hernández-Lerma, O., Vega-Amaya, O. and Carrasco, G. (1999). Sample-path optimality and varianceminimization of average cost Markov control processes. SIAM J. Control Optimization 38, 79-93.

[7] Hilgert, N. and Hernández-Lerma, O. (2003). Bias optimality versus strong 0-discount optimality in Markov control processes with unbounded costs. Acta Appl. Math. 77, 215-235.

[8] Jarner, S. F. and Roberts, G. O. (2002). Polynomial convergence rates of Markov chains. Ann. Appl. Prob. 12, 224-247.

[9] LÁNSKÁ, V. (1986). A note on estimation in controlled diffusion processes. Kybernetika 22, 133-141.

[10] Luque-VÁsquez, F. And Hernández-Lerma, O. (1999). Semi-Markov control models with average costs. Appl. Math. 26, 315-331.

[11] MandL, P. (1971). On the variance in controlled Markov chains. Kybernetika 7, 1-12.

[12] MandL, P. (1973). A connection between controlled Markov chains and martingales. Kybernetika 9, $237-241$. 
[13] Mandl, P. (1974). Estimation and control in Markov chains. Adv. Appl. Prob. 6, 40-60.

[14] MandL, P. (1974). On the asymptotic normality of the reward in a controlled Markov chain. In Progress in Statistics (European Meeting of Statisticians, Budapest, 1972), Vol. II, Colloquia Mathematica Societatis János Bolyai Vol. 9, North-Holland, Amsterdam, pp. 499-505.

[15] Mandl, P. and Laušmanová, M. (1991). Two extensions of asymptotic methods in controlled Markov chains. Ann. Operat. Res. 28, 67-79.

[16] Mendoza-PÉrez, A. (2008). Asymptotic normality of average cost Markov control processes. Morfismos 12, $33-52$.

[17] Mendoza-PÉrez, A. (2008). Pathwise average reward Markov control processes. Doctoral Thesis, CINVES TAV-IPN. Available at http://www.math.cinvestav.mx/ohernand_students.

[18] Mendoza-Pérez, A. F. And Hernández-Lerma, O. (2010). Markov control processes with pathwise constraints. Math. Meth. Operat. Res. 71, 477-502.

[19] Meyn, S. P. And Tweedie, R. L. (1993). Markov Chains and Stochastic Stability. Springer, London.

[20] Prieto-Rumeau, T. and Hernández-Lerma, O. (2009). Variance minimization and the overtaking optimality approach to continuous-time controlled Markov chains. Math. Meth. Operat. Res. 70, 527-540.

[21] Puterman, M. L. (1994). Markov Decision Processes: Discrete Stochastic Dynamic Programming. John Wiley, New York.

[22] Vega-Amaya, O. (1998). Markov control processes in Borel spaces: undiscounted criteria. Doctoral Thesis, UAM-Iztapalapa (in Spanish).

[23] Zhu, Q. X. AND Guo, X. P. (2007). Markov decision processes with variance minimization: a new condition and approach. Stoch. Anal. Appl. 25, 577-592. 